\title{
Focal mechanisms overview
}

\author{
Ahmed R. Khalil ${ }^{1}$ (D) Nassir S. Al-Arifi ${ }^{2}$ \\ Received: 11 April 2018 / Accepted: 24 October 2018 / Published online: 19 November 2018 \\ (C) The Author(s) 2018
}

\begin{abstract}
A new computer application is created to use graphical representation of seismological data sets namely focal mechanism data to help seismologist to minimize complexities, recognize trends, discover data patterns and finally to develop the hypotheses. These graphical representation views are widely used by seismologist to drive information out of its long datasets. Using visual studio dot net 2012 to create a windows based software tools for focal mechanism datasets analysis using triangle diagrams. Another analysis tool is bar graph using basics of rose diagrams to plot the distribution of data frequency of each class. The third analysis tool included is a 4D plot of the distribution of earthquake events versus its depths and magnitude. A new classification of plotting double-couple earthquake focal mechanisms on the ternary diagram is defined. The application software has many advantages like automatic writing the analysis reports which contain all calculated information represented in the software graphs. This report helps for advanced processing. Automatic prototype analysis reporting can be considered as the first step (milestone) for applying expert systems in focal mechanism analysis tools. The application program interface (API) are designed to be user friendly and use interactive screens to customize the analysis.
\end{abstract}

Keywords Software tools $\cdot$ Focal mechanism analysis $\cdot$ Rose diagram $\cdot$ Geophysical data processing $\cdot$ Ternary diagrams

\section{Introduction}

Seismologic datasets are typically large and compiled into long tables or large databases, but pure numerical information can be

\footnotetext{
Highlights

1. Analysis tools integrated in new software for graphical representation of focal mechanism datasets.

2. Analysis tools are ternary diagrams, bar graph using basics of rose diagrams and a 4D plot of the earthquakes locations versus depths and magnitude.

3. Some advantages: automatic analysis report, user friendly and interactive screens.
}

Communicated by: H. A. Babaie

Electronic supplementary material The online version of this article (https://doi.org/10.1007/s12145-018-0367-1) contains supplementary material, which is available to authorized users.

Ahmed R. Khalil

arkhali144@gmail.com; a.khalil@su.edu.sa

Nassir S. Al-Arifi

nalarifi@ksu.edu.sa

1 College of Computing and Information Technology, Shaqra

University, Shaqra, Saudi Arabia

2 College of science, King Saud University, Rayadh, Saudi Arabia difficult to understand and interpret. A lot of separate scattered software exists in the literature like "OpenRose" version 0.01 which is written in C\#. NET, last updated on 2009-09-13 (https://openrose.soft112.com/, 2017). Some software are licensed or developed for solving specific problem, or developed for a special project. Another problem is that these different software are developed on different platforms such as Unix, Xenix, Linex, Dos, windows or on Apple Operating system, therefore, scientists should have the same environment to use the software. Other cases, seismologist (users) may create their own libraries or functions to process their data, in these cases other scientists (new users) should have the same compilers (Fortran 77 or C\# compilers) and should understand clearly what are the data types or format needed to be processed. A third problem is the long list of old input data stored on different media which should be read and collected in a specific digital format to be ready for analysis software. Many efforts have been done to calculate quantitative amount of data as a preliminary step for seismic focal mechanism analysis. For example, in an effort to improve data services, the National Earthquake Information Center cooperate with the Incorporated Research Institutions for Seismology Data Management Center (IRIS DMC), to produce rapid estimates of the seismic moment tensor for a large earthquakes data set (Sipkin 1994). Also, Isacks and Molnar 1971, had a set of 


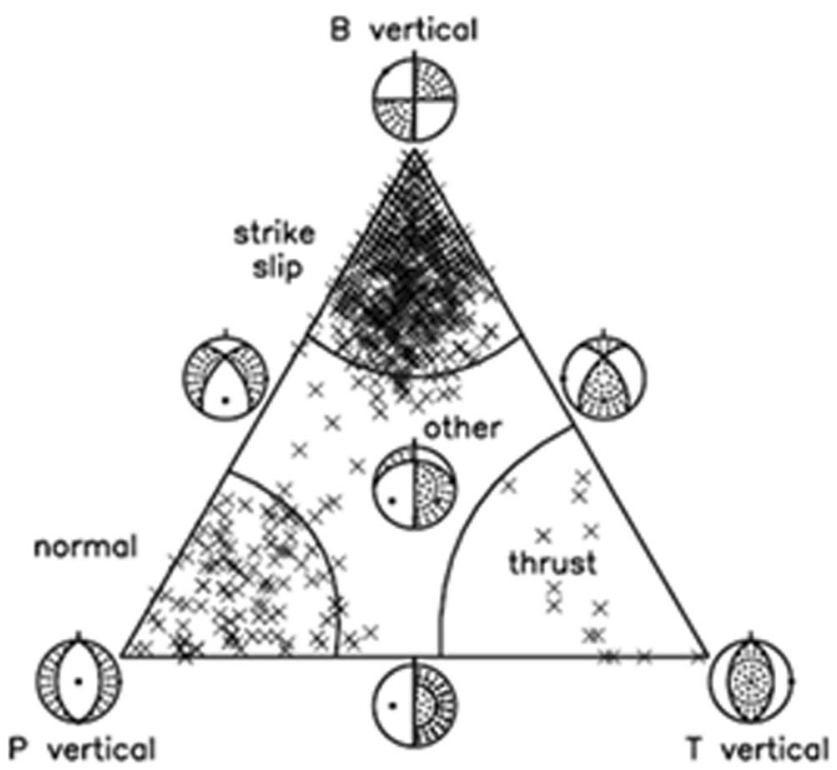

Fig. 1 Cliff Frohlich seismic zones ((Frohlich 1992)

204 reliable focal-mechanism solutions for analysis to support their ideas (Isacks and Molnar 1971).
A region-by-region analysis of 204 reliable focalmechanism solutions for deep and intermediate-depth earthquakes strongly supports the idea that portions.

This drives scientists to use graphical representation to minimize complexities, recognize trends and discover data patterns to develop the hypotheses.

To solve all the above problems using open source new application software developed to be user friendly reading old data with almost all format and working on all operating system environments. An example is the DurovPwin software developed to solve these problems in the field of groundwater major dissolved constituents and hydrochemical evolution (Al-Bassam and Khalil 2012). The DurovPwin software, for example, not only solve the researcher problems but also improves the original Durov diagram giving extended Durov diagram and adding many new analysis features.

In this paper, some technical problems in the geophysical field were studied carefully and considered during the implementation of some helpful tools in the field of seismology specially, focal mechanism aspects. This application integrates the ternary diagram and also adding new zones definition, and

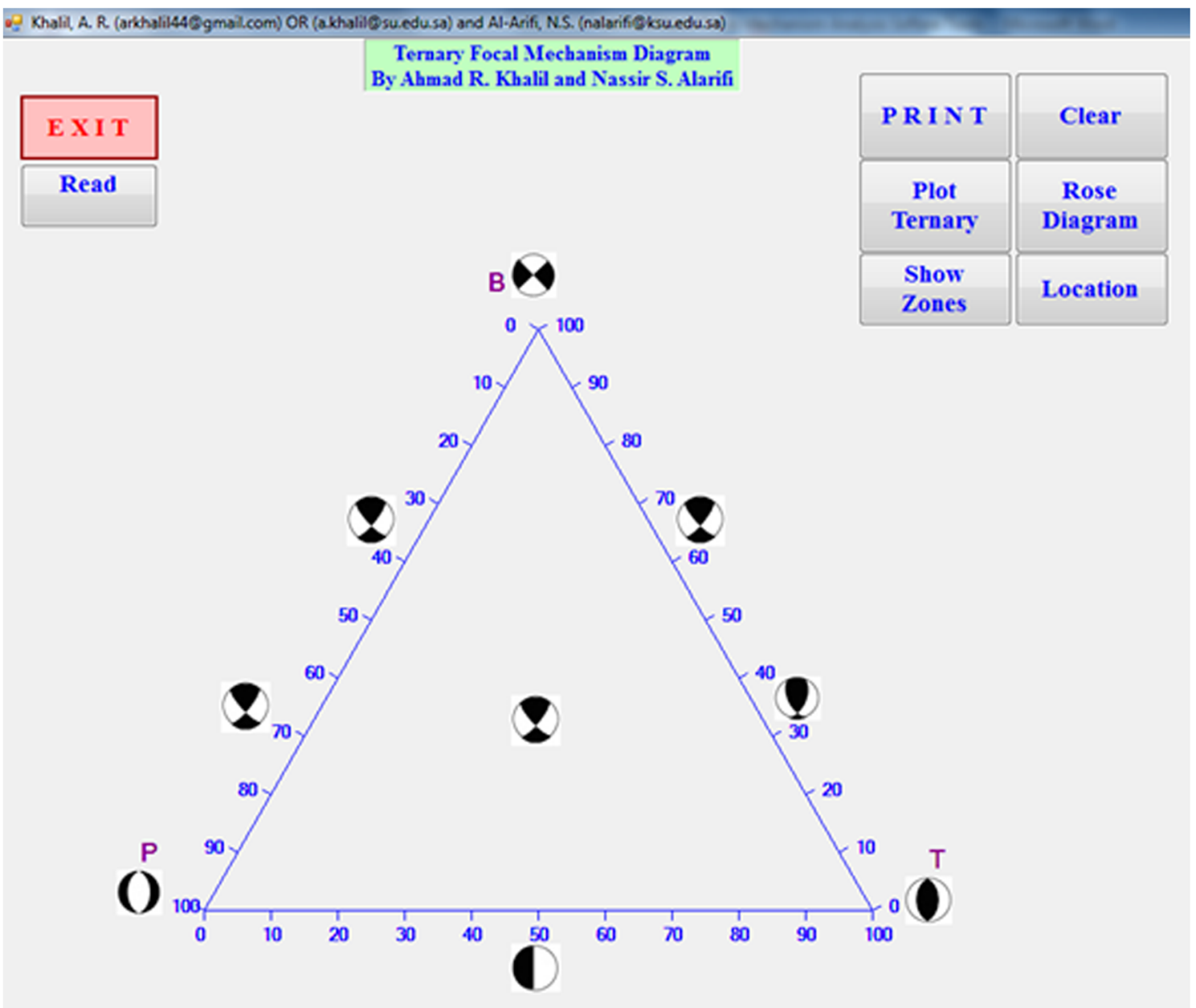

Fig. 2 Opening window of the TFMtools software 
Fig. 3 Application procedure of the TFMtools

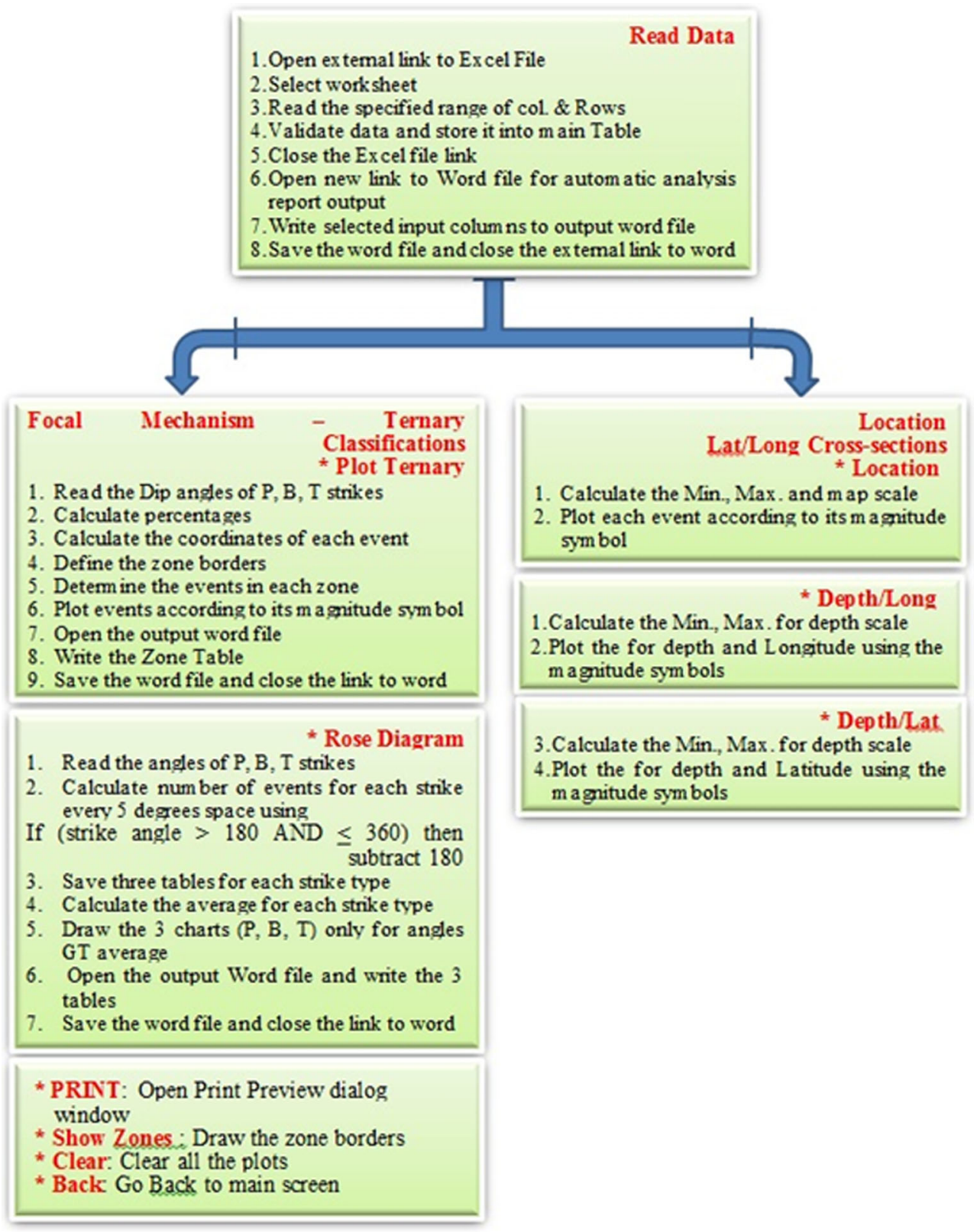

the bar graph representing the rose diagram while giving the option of plotting the symbols according to its magnitude. The source input data is Microsoft excel file which is the simplest form of data, this facilitate the collection of data from any catalogue all over the world. USGS catalogue is widely used for focal mechanism analysis using the FPFIT program (Reasenberg and Oppenheimer 1985) and will output multiple fault plane solutions. Using the MKTABLE, a FORTRAN program provided by the USGS to view the focal mechanism profile. This is a difficult process especially in case of a large number of earthquakes (using quantitative methods). Therefore, ternary diagrams should be used to categorize the different types of focal mechanisms generated.

\section{Prototype implementation of focal mechanisms analysis tools}

The new software presented here integrate three analysis tools combined together is called Ternary Focal mechanism (TFMtools) software tools. The first tool is the method of evaluating the distribution of the focal mechanism orientations of groups of earthquakes and displays the data on the triangle diagram. The second tool is the histogram plot which displays directional data and the frequency of each class known as rose diagram. The last tool is plotting the earthquakes distribution on Latitude and Longitude versus its depth which shows many 


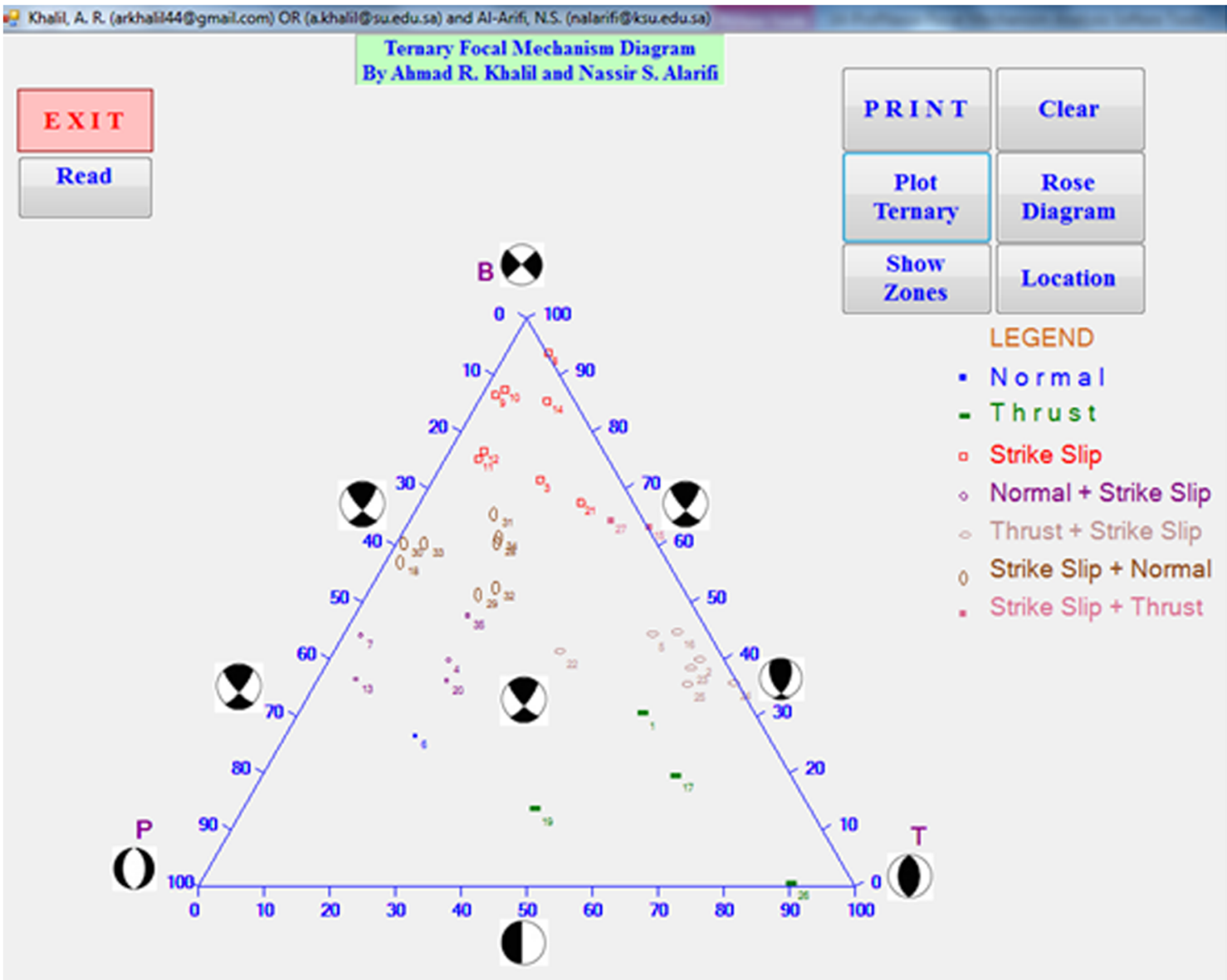

Fig. 4 Distribution of earthquake focal mechanisms

analytical facts. A quick description of these three tools is given below.

\section{Triangle diagram}

Sedimentology scientists commonly use graphical representations to reduce complexities, recognize trends and patterns in the data, and develop hypotheses. The most common methods used by sedimentologists is to plot the basic gravel, sand, silt, and clay percentages on equilateral triangular diagrams. This present data simplify and facilitates rapid classification of sediments and comparison of samples (Poppea and Eliasonb 2008). Another example for using the triangle diagram for data analysis is the research by Lawrence and others where they wrote four computer programs to facilitate analysis of sediment grain-size data (Lawrence et al. 2010). Also in seismology, Henri s. Swolfs (Swolfs 1984) introduces the triangle diagram as a new graphical method that facilitates the representation and interpretation of the generally anisotropic in situ- states-of stress measured in rocks. The purpose of the method is to clearly and easily display these stress states, each measured in terms of three values, in two-dimensional space; this is done by projecting three-dimensional stress space onto a triangular-coordinate (ternary) diagram and plotting the measured stress states as points (Henri s. Swolfs 1984). Cliff Frohlich introduced the ternary method for displaying focal mechanism by using each vertex to represent Normal, Thrust, and Strike-Slip focal mechanisms (Frohlich 1992). So, ternary diagrams are graphs used to plot plotting double-couple earthquake focal mechanisms using the three vertices to corresponds 'pure' strike-slip, normal, and thrust mechanisms (Frohlich 1992, 1996; Frohlich and Apperson 1992). Also, Frohlich states that triangle diagrams are simply a quantitative graphical method for using the dip angles of T, B and P axes for displaying focal mechanisms. Frolich shows how to display the distribution of focal mechanism orientations of groups of earthquakes on a triangle diagram using quantitative methods (Frohlich 2001). Some general purpose software was done to plot ternary diagram using Microsoft Excel, but it only show the diagram without any more comments or analysis on the result shown.

Cliff Frohlich divides the seismic zones into 4 divisions on the ternary diagram (Frohlich 1992). These four regions are thrust, strike-slip, and normal where T, B, P axes lie within 


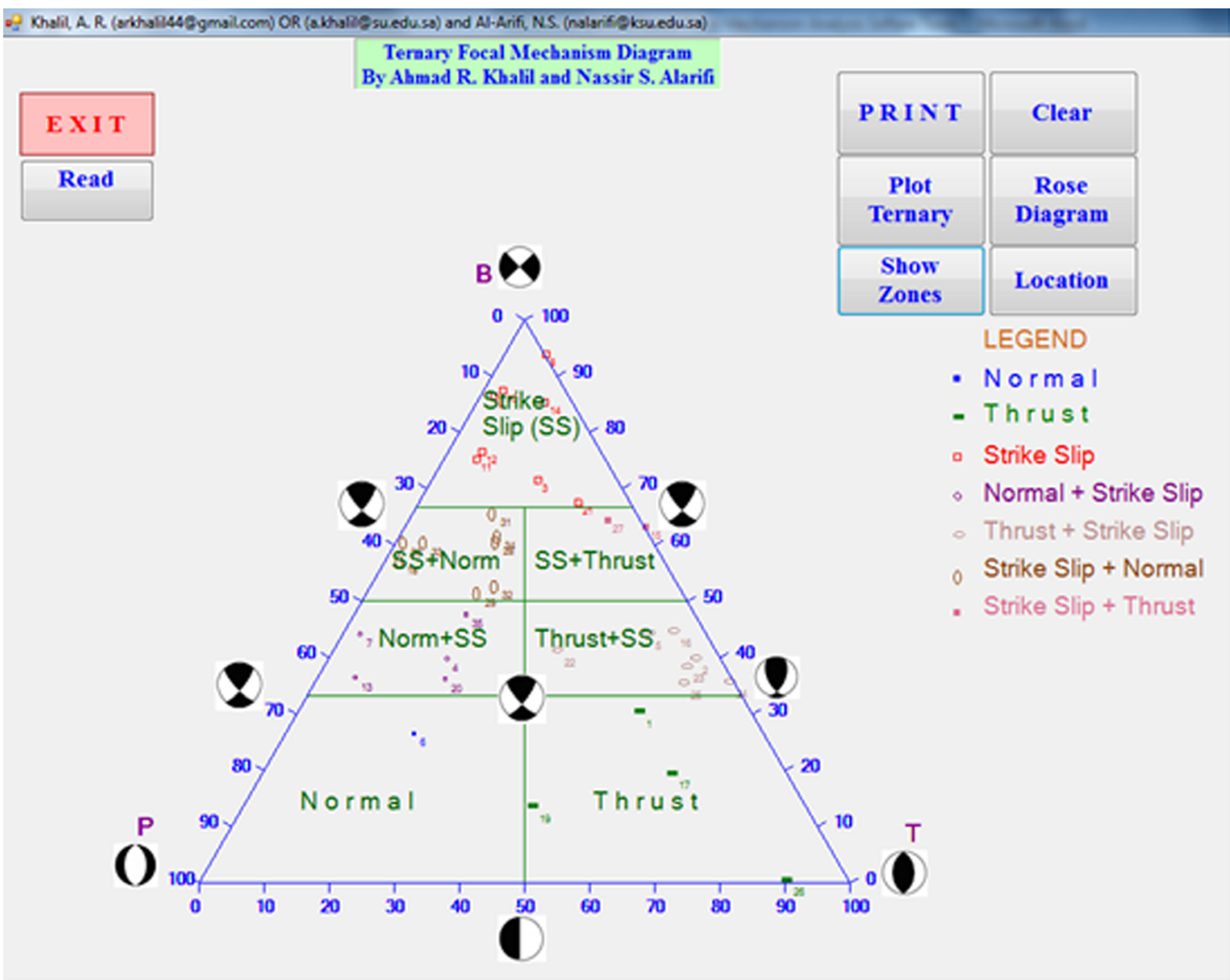

Fig. 5 Zones classifying the distribution of earthquake focal mechanisms

$40^{\circ}, 30^{\circ}$, and $30^{\circ}$ of the vertical, respectively. The forth region is the region for all the remaining mechanisms and classified as "others" as shown in Fig. 1. The same zone definitions are used by Borges et al. (Borges et al. 2001).

\section{Histogram plot}

A histogram plot which displays directional data and the frequency of each class is called rose diagram when the histogram is on circular shape. Rose diagrams are commonly used in sedimentary geology to display palaeo current data, or the orientation of particles, and in structural geology to plot the orientation of joints and dykes. Also, wind directions and frequencies can also be plotted on rose diagrams. Histogram plot are useful for showing azimuthal (directional) data. Generally, any dataset consisting of lots of measurements of direction or orientation could be visualized this way.

\section{Earthquake location-depth diagram}

Another analysis method of long tables of geophysical data or seismic hazards data is Location depth diagram. Generally, almost all researches in this field present their long list of earthquakes on maps or on $x-y$ plot of latitude and longitude to visualize the data relative to its location. Recently, researcher improves the Lat. vs. Long (location) plot to be 3D plot adding depth as the third dimension. Integrating these three $\mathrm{x}$ y plot on one graph gives a great analysis force to visualize the cross section of earthquake locations with depth. In the application proposed, the magnitude representation of the earthquakes is added to visualize a forth parameter.

\section{Description of application proposed}

The main goals of the application software are to provide visualization of graphical representation of focal mechanism data sets, creating analysis report automatically, also be user friendly, and many other advantages. The application contains interface windows which designed to facilitate execution, allowing users to select options primarily with minimum mouse-click, or through interactive dialogue boxes. Also the designed screens are almost similar to the most commonly used and all dialogues are of Microsoft Dot net dialogues. 


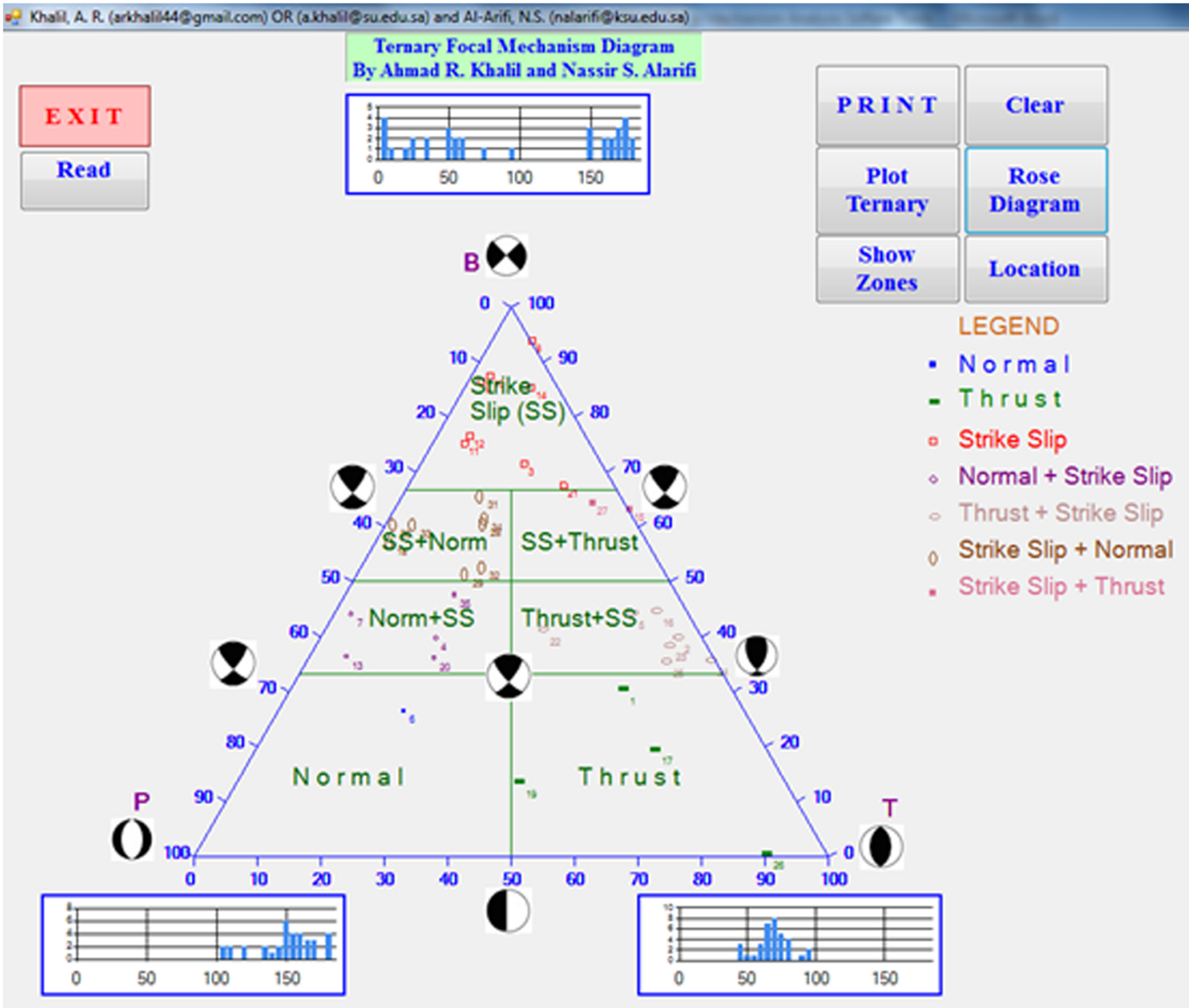

Fig. 6 Histogram distribution, seismic zones, and distribution of earthquake according to its focal mechanism all integrated in one screen while all details are written in the automatic analysis report

Therefore the new application looks familiar and is user friendly.

\section{Software procedure}

The Ternary Focal Mechanism tools (TFMtools) software starts from the main screen to read data, then an open file dialogue starts to find out the user data file. The read process starts and a data validation to find out zeros or empty data cells where it must be a numeric value. When finish reading data, a message showing the number of columns read and end of reading data is displayed. The software has two main screens, first is the ternary diagram where the distribution of the focal mechanisms are plotted and the rose diagram represented by bar graph on the three vertices of the triangle. The first screen also contains a button to show the different zones in the triangle, and another button to open a print dialogue. Figure 2 shows the opening screen with all the buttons and the ternary diagram with the symbols of beach ball representing the different focal mechanisms for any earthquake.
The second screen contains 4D graph represented by three $\mathrm{X}-\mathrm{Y}$ plots relations, namely Latitude versus Longitude, Latitude versus depth, and Longitude versus depth. The forth parameter is an option to represent the magnitude.

The complete application software procedure is shown in Fig. 3. The first button is "Read Data" which contains 8 actions, starting from opening the link to the specified Excel file and the default worksheet, then the selected columns and rows. While reading the data, it checks for zeros of important data items which may produce overflow error, then close the file link. The second main function is to start writing the automatic analysis report which the software will save all the analyzed results, therefore, it will open an regular Open File dialogue, and write the raw data which will be used throughout the software. This is to give the user that all the results given in the report starts from these source raw data, so any wrong number in the given input data will show up in the beginning of the report. As shown from Fig. 3, there are two main paths, one for X-Y plot events (sized by magnitude is option) and the other Path is to plot the ternary, rose diagram, and show the seismic zones. 


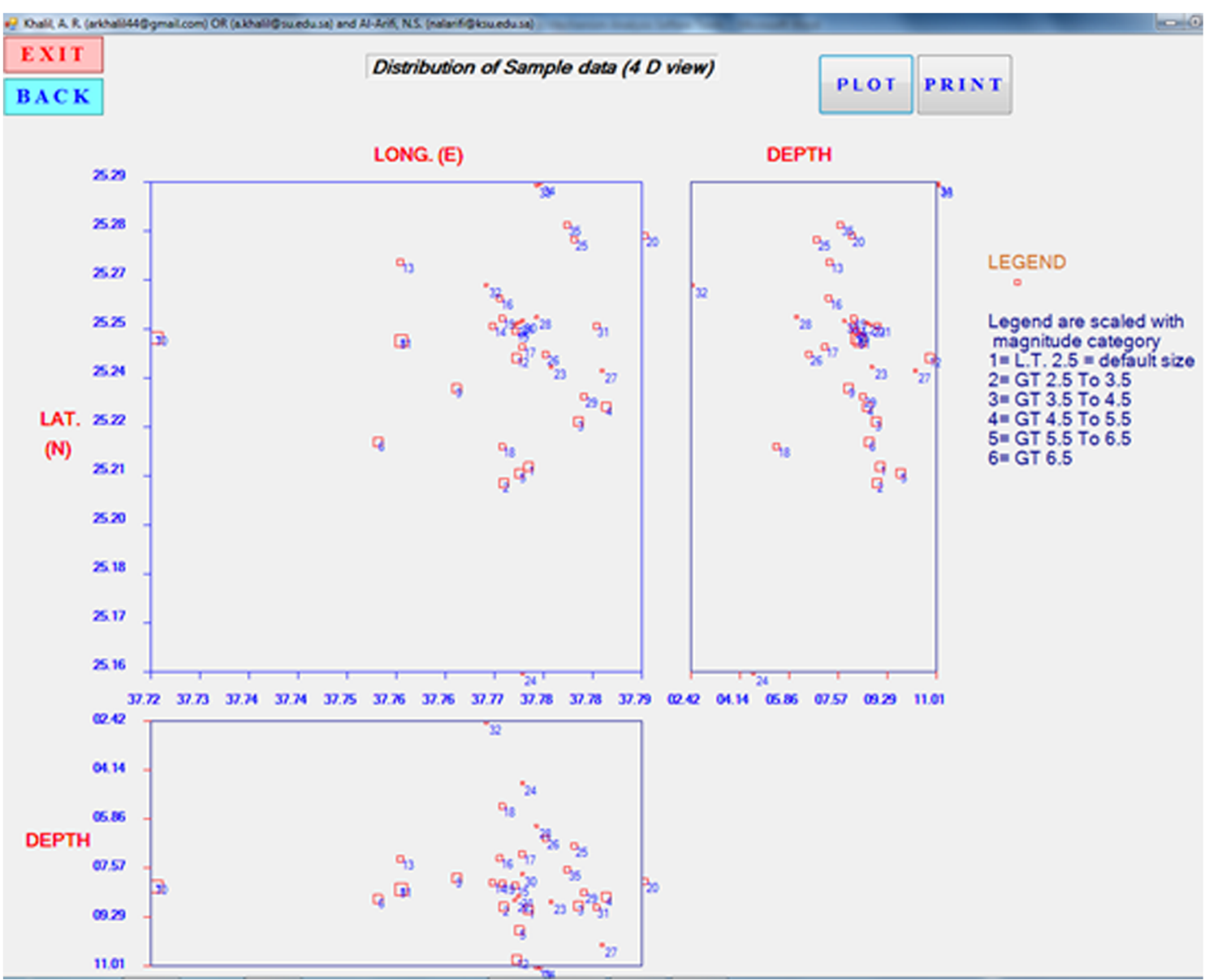

Fig. 7 The second window of the TFMtools software which shows the distributions of the earthquakes on a map (Lat. and Long.) with two cross-sections of the depth

\section{Results and discussions}

A sample data file is used for a location between latitude: $25.156 \mathrm{~N}$ to $25.2935 \mathrm{~N}$ and longitude between 37.724 to 37.7919E Saudi Arabia. The depth is found to be between 2.42 to $11.01 \mathrm{KM}$. After reading data from the selected file, the software asked for the output file and give an end reading source data file message. Pressing the "Plot Ternary button, all events are displayed according to its dip angles. The code defines strike, dip and rake according to the conventions set forth by Aki and Richards (1980), Quantitative Seismology, Vol. 1 and extend these classifications by four more zones. Figure 4 shows the result with the legend to show the classifications.

On the plot, user may show the different zones to easy classify the seismic events, this can be done by pressing "Show Zones" button, see Fig. 5. The last information which can be deduced from the same data is the concentration of the directional data in categories of $5^{\circ}$ which can be seen also in the same screen as bar graph on each triangle vertex $(B, P, T)$, Fig. 6 shows these rose diagram like after pressing the "Rose
Diagram" button. Note that all these classification shown on figures are also listed as tables in the automatic analysis report.

The second window is the $\mathrm{X}-\mathrm{Y}$ plot of latitude, Longitude, and depth. These plots are shown by pressing the Location button from starting screen. This will open the second screen with empty three areas to show these relations. Pressing "plot" button, opens dialogue to write the suitable title of the graph, then all the three relations are plotted.

Figure 7, show the final distribution of the seismic earthquakes distributed on a map like i.e. latitude-Longitude distributions. In addition two cross-sections are shown with depth to show a 3D view. From this window the earthquakes can be classified to be shallow or deep events. Then representing the magnitude scale symbols gives the 4D representation.

The automatic analysis report is shown in the appendix.

\section{Conclusions}

The new implementation of the software used for focal mechanism analysis provides many advantages using data 
graphical representation approach. First, it integrates several tools into single software. Second advantage, it produce analytic reports automatically. The ternary diagram improves the data classification into seven divisions. Dataset from Saudi Arabia is used to confirm the effectiveness of the application and time spent to write code.

Publisher's Note Springer Nature remains neutral with regard to jurisdictional claims in published maps and institutional affiliations.

Open Access This article is distributed under the terms of the Creative Commons Attribution 4.0 International License (http:// creativecommons.org/licenses/by/4.0/), which permits unrestricted use, distribution, and reproduction in any medium, provided you give appropriate credit to the original author(s) and the source, provide a link to the Creative Commons license, and indicate if changes were made.

\section{References}

Aki K, Richard, PG (1980) Quantitative Seismology, W. H. Freeman, San Francisco

Al-Bassam AM, Khalil AR (2012) DurovPwin: a new version to plot the expanded Durov diagram for hydro-chemical data analysis. Comput Geosci 21:1-6

Borges JF, Fitas AJS, Bezzeghoud M, Teves-Costa P (2001) Seismotectonics of Portugal and its adjacent Atlantic area. Tectonophysics 331:373-387

Frohlich C (1992) Triangle diagrams: ternary graphs to display similarity and diversity of earthquake focal mechanisms, Physics of the Earth and Planetary Interiors, 75, 193-198, Elsewer Science Pubhshers B $\mathrm{V}$, Amsterdam

Frohlich C (1996) Cliff's nodes concerning plotting nodal lines for P, Sh, and Sv, seism. Res Lett 67:16-24

Frohlich C (2001) Display and quantitative assessment of distributions of earthquake focal mechanisms. Geophys J Int 144:300-308

Frohlich C, Apperson KD (1992) Earthquake focal mechanisms, moment tensors and the consistency of seismic activity near plate boundaries. Tectonics 11:279-296

https://openrose.soft112.com/ (2017) 09:28, Simple open source Windows C\# .NET application to create rose diagrams, last updated 2009-09-13

Isacks B, Molnar P (1971) Distribution of stresses in the descending lithosphere from a global survey of focal-mechanism solutions of mantle earthquakes. Rev Geophys Space Phys 9:103-174

Lawrence J. Poppe, Andrew H. Eliason, and Kathrine Y. McMuller (2010) A versatile suite of laboratorynonspecific software for processing sediment grain-size data, 2nd Joint Federal Interagency Conference, Las Vegas

Poppe LJ, Eliason AH (2008) Short note: a visual basic programto plot sediment grain-size data on ternary diagrams. Comput Geosci 34: 561-565

Reasenberg P, Oppenheimer D (1985) FPFIT, FPPLOT and FPPAGE: Fortran computer programs for calculating and displaying earthquake fault-plane solutions, U.S. Geological Survey, Open-File Report No. 85-739, 109

Sipkin SA (1994) Rapid determination of global moment-tensor solutions. Geophys Res Lett 21:1667-1670

Swolfs HS (1984) The Triangular Stress Diagram A Graphical Representation of Crustal Stress Measurements, United States government printing office, Washington, (Geological Survey Professional Paper; 1291), 19 